\title{
Homozygosity mapping in a family presenting with schizophrenia, epilepsy and hearing impairment
}

\author{
Helen M Knight ${ }^{*, 1,2}$, Alan Maclean ${ }^{2}$, Muhammad Irfan ${ }^{3}$, Farooq Naeem ${ }^{4}$, Stephen Cass ${ }^{2}$, \\ Benjamin S Pickard ${ }^{2}$, Walter J Muir ${ }^{1}$, Douglas HR Blackwood ${ }^{1}$ and Muhammad Ayub
}

\begin{abstract}
${ }^{1}$ Division of Psychiatry, University of Edinburgh, Royal Edinburgh Hospital, Edinburgh, UK; ${ }^{2}$ Medical Genetics, University of Edinburgh, School of Clinical and Molecular Medicine, Molecular Medicine Centre, Western General Hospital, Edinburgh, UK; ${ }^{3}$ Lahore Institute of Research and Development, Lahore, Pakistan; ${ }^{4}$ Fatima Jinnah Medical College, Lahore, Pakistan; ${ }^{5}$ St Lukes Hospital, Middlesborough, UK
\end{abstract}

Homozygosity mapping within consanguineous families is a powerful method of localising genes for autosomal recessive disease. We investigated a family from Punjab, Pakistan, a region where consanguineous marriages are frequent. The parents have no detectable clinical disorders. However, five out of six children present with schizophrenia, epilepsy or hearing impairment either alone or in combination. This unusual phenotype in several offspring of first cousins is strongly suggestive of a rare, Mendelian recessive disorder. Two genome-wide scans initially using low-density microsatellites, and subsequently high-density SNP markers were used to map homozygous-by-descent regions in affected individuals. Candidate genes within these loci were subsequently screened for mutations. Homozygosity analysis and inbreeding coefficients were investigated to give an estimate of consanguinity. Two putative disease loci were mapped to $22 q 12.3-q 13.3$ and 2 p24.3. The candidate locus on chromosome 2 p24 overlaps with a deafness locus, DFNB47, linked to autosomal recessive hearing impairment, while positive findings reported for affective psychosis and schizophrenia cluster in a region of 4-5 cM on 22q13.1 within our second candidate locus. Sequence analysis of three candidate genes (KCNF1 (2p); ATF4, CACNG2 (22q)) did not reveal any exonic mutations. Inbreeding coefficients calculated for each family member support a very high degree of ancestral and recent inbreeding. The screening of other candidate genes located within these newly identified disease intervals on Chr2p24.3 and 22q12.3-q13.3 may lead to the discovery of causative variants, and consequent disrupted molecular pathways associated with this rare phenotype. European Journal of Human Genetics (2008) 16, 750-758; doi:10.1038/ejhg.2008.11; published online 5 March 2008

Keywords: hearing impairment; epilepsy; schizophrenia; homozygosity mapping; chromosome 2; chromosome 22

\section{Introduction}

Populations that are genetically isolated or have a high frequency of inbreeding tend to show an increased

*Correspondence: HM Knight, Medical Genetics, University of Edinburgh School of Clinical and Molecular Medicine, Molecular Medicine Centre, Western General Hospital, Crewe Road, Edinburgh. EH4 2XU, Scotland. Tel: + 44131651 1056; Fax: + 44131651 1059;

E-mail: s0343996@sms.ed.ac.uk

Received 25 September 2007; revised 19 December 2007; accepted 6 January 2008; published online 5 March 2008 prevalence of recessive disorders. Historically, the study of inbred populations has been highly successful in the fine mapping of recessive traits. The effect of consanguinity reduces allelic and non-allelic heterogeneity and potentially increases linkage disequilibrium and hence the power to detect associations between genetic markers and disease. ${ }^{1}$ Likewise, mapping homozygous regions in affected individuals in consanguineous families is a powerful method of localising autosomal recessive genes. This technique known as homozygosity or autozygosity 
mapping assumes that affected offspring co-inherit two copies of a disease-related chromosomal segment from a common ancestor. Such positional cloning of recessive genes in families with rare genetic variants can provide valuable insight into the localisation and identity of genetic susceptibility factors involved in the common types of disease with similar clinical features. ${ }^{2}$

The more closely related the parents are, the greater proportion of their offspring's genome is expected to be homozygous. For example, offspring of first-cousin marriages are expected to have $1 / 16(6.25 \%)$ of their genome homozygous and for second cousins the fraction is $1 / 64$ $(1.56 \%)$. Formally, this figure, which represents the proportion of homozygous autosomal genome, is known as the inbreeding coefficient $(F)$. It can be used to estimate parental relationships and highlight additional ancestral inbreeding loops. However, the greater the expected homozygosity in offspring, the greater the likelihood of identifying shared homozygous-by-descent (HBD) intervals in affected siblings who are unrelated to the 'disease'causing locus.

The family investigated comes from North Punjab, Pakistan - a region where consanguineous marriages are frequent. The parents are first cousins and there is additional (remote) consanguinity in the ancestral kindred. The parents themselves have no detectable clinical disorders; however, five out of their six children present with schizophrenia, epilepsy or sensori-neural hearing impairment or a combination of all the three (Figure 1). This unusual phenotype in several offsprings of a cousin marriage is strongly suggestive of a rare, Mendelian recessive disorder, although other forms of inheritance cannot be dismissed. This family, therefore, presents with a unique opportunity to identify one or several autosomal recessive genes for these conditions.

Hereditary hearing impairment is a highly genetic heterogenous condition subdivided into syndromic and non-syndromic forms. A total of $77 \%$ of non-syndromic cases show autosomal recessive inheritance and commonly give rise to sensori-neural defects. ${ }^{3}$ Approximately 50 loci have been linked to recessive non-syndromic hearing impairment NSHI, the majority of which were mapped in large consanguineous pedigrees. ${ }^{4}$ Mutations in over 20 genes have subsequently been identified and typically cause disruption of the molecular mechanisms that underlie sensory processing in hair cells of the cochlea. ${ }^{5}$

Epilepsy can be characterized by partial or generalized seizures depending on the pattern and localization of ictal activity within the brain. Further sub-classification of forms into specific syndromes involves evaluation of age of onset, clinical and electroencephalographic features. Both monogenic and polygenic inheritance has been described, indicating variation in both genetic factors and mode of inheritance. Linkage mapping in pedigrees with monogenic Mendelian epilepsy has revealed several causative genes encoding ion channel subunits. Moreover, variants in two genes, a T-type calcium channel subunit and $\mathrm{GABA}_{\mathrm{A}} \delta$-receptor subunit have been implicated by electrophysiological studies for some forms of complex epilepsy (reviewed in ref. ${ }^{6}$ ).

Schizophrenia is a debilitating disease characterized by disordered moods, beliefs, perceptions and movements often accompanied by cognitive impairment. Different genetic factors are postulated to contribute to schizophrenia, and environmental factors are acknowledged to play a role. Investigating genetic contributions to schizophrenia is complicated by the likely presence of variable phenotypic presentation, incomplete penetrance and both locus and allelic heterogeneity. It is still a matter of debate whether, in addition to environmental interaction, disease most frequently results from the interaction of many genes of small effect (quantitative trait locus theory) or many single gene defects of major effect (oligogenic model). ${ }^{7,8}$ Given the uncertainties concerning the mode of inheritance of schizophrenia, the study of rare, even singular, families may provide important clues about the genetic aetiololgy of the illness.

In this study, homozygosity mapping was performed by means of two genome-wide scans with the aim to fine map HBD regions potentially containing recessive disease genes. To measure the extent of consanguinity, inbreeding coefficients were estimated for each family member, and

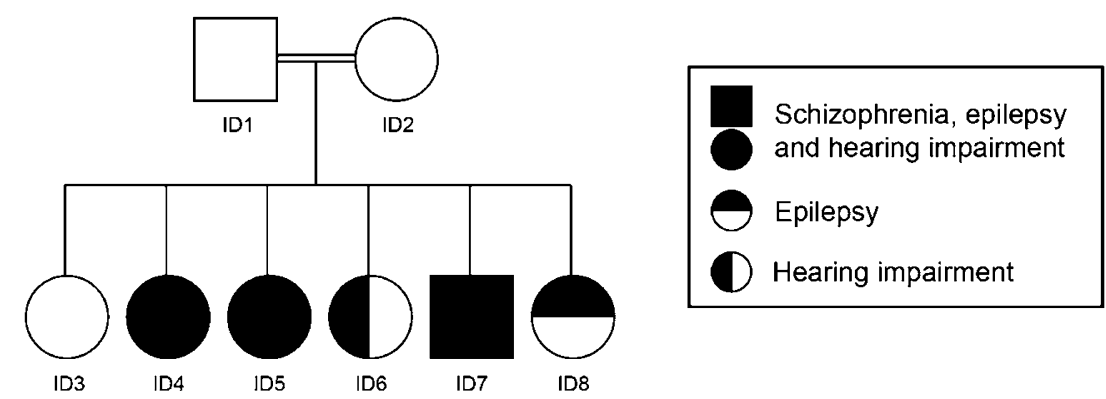

Figure 1 Diagram of pedigree. Open shapes represent non-affected individuals. Filled shapes denote siblings with schizophrenia, epilepsy and hearing impairment. Half-filled circles represent epilepsy or hearing impairment. Double lines between individuals denote first cousins. 
detailed analysis of autosomal homozygous segments was conducted. Finally, three promising candidate genes, KCNF1, ATF4 and CACNG2, located within the two newly identified HBD loci were screened for mutations.

\section{Methods and materials Clinical data}

A family (Figure 1) from a small village in north-west Punjab where there is a strong tradition of marriages between cousins was identified by one of the authors (MA), an experienced psychiatrist who speaks the local language. Fully informed written consent was attained from each member of the family. The study was approved by the appropriate UK and Pakistani ethics of research committees. Clinical information was gathered through multiple interviews with all members of the family and inspection of hospital records of inpatient admissions. The parents gave no history of still births or miscarriages. Physical examination of all members revealed no signs of systemic illness or dysmorphic features. Investigations confirmed normal haematology and liver and renal function. Computed tomography brain scans of two affected siblings indicated no structural changes, and electroencephalography showed only minor non-specific alterations in the same two individuals. The diagnosis of schizophrenia was made according to ICD-10 criteria based on direct interviews using the Schedule of Affective Disorders and Schizophrenia, and information from informants and hospital care records. Audiometry confirmed sensorineural hearing impairment.

Cognitive function was assessed by a clinical psychologist, and schools were approached for information about educational performance. The affected siblings had normal physical and cognitive development with normal performance in school until around the age of 8 years, when they presented with partial complex seizures sometimes leading to generalized seizures. At approximately the same age, these individuals developed tinnitus and sensori-neural hearing loss that became progressively more profound during adolescence, leading to severe loss of hearing by the age of 20. Three siblings had experienced psychotic episodes by the age of 25 years and met ICD 10 criteria for schizophrenia. Symptoms included auditory hallucinations and delusions of reference and persecution with no affective component. The psychosis is intermittent, with good recovery between the episodes that have responded well to treatment with antipsychotic medication. The oldest sibling, now in late forties, is unaffected. Three of the siblings who are over 30 years old have all three components of the phenotype. One of the younger siblings has only impaired hearing and one has epilepsy, but neither of these siblings who are in their twenties have had symptoms of psychosis although they remain at the risk of developing symptoms. The oldest of the affected siblings received in-patient treatment in a psychiatric unit where she responded to antipsychotic medication, but later relapsed because of poor compliance. In all of the affected siblings, epilepsy has responded to carbamazepine. Between episodes of psychosis, the social and occupational functioning of the affected siblings is well preserved.

\section{Genotyping}

Genomic DNA was extracted using standard methods by Genovar Diagnostics. The initial genome-wide scan for linkage employed 396 highly polymorphic microsatellite markers (ABIPRISM linkage set, MD10 version 2), with an average spacing of $\sim 10 \mathrm{cM}$. Markers were labelled with fluorescent dyes and assayed in 28 multiplexed panels. PCR was performed according to the manufacturer's specifications using a peltier thermal cycler (PTC-225, DNA Engine tetrad). PCR products were run on an ABI 3730 DNA sequencer. Genotypes were called using GeneMapper software version 3.0 and electropherograms were examined visually. Nineteen additional markers used for fine mapping in the region of 22q12.3-13-3 were selected on the basis of their heterozygosity and location, manufactured by Invitrogen and genotyped as described above. Primer sequences are available on request. Marker information and chromosomal physical distances were obtained from UCSC Genome Browser v52 website. Allele frequencies were obtained from CEPH Genotype database browser V2.1 site (http://www.cephb.fr/cephdb/php/eng). A second genome-wide scan employed 5858 biallelic SNPs typed at Illumina Inc., San Diego, USA, using proprietorial bead-array technology (Linkage IV panel). Marker spacing was $\sim 480 \mathrm{~kb}(0.6 \mathrm{cM})$ and locus success rate was $99.2 \%$. SNP data was analysed and checked for potential genotyping errors both by visual examination and by PLINK v0.99 Mendel error test (http://pngu.mgh.harvard.edu/ purcell/ plink/). LOD scores were calculated using the 'MLINK' set of programmes. ${ }^{9}$ A recessive model of inheritance and full penetrance was assumed.

\section{Inbreeding coefficients and genome-wide analysis autozygosity}

Inbreeding coefficients $(F)$ were estimated using FEstim. ${ }^{10,11}$ Minor allele frequencies as determined for both the Caucasian CEPH and Asian populations (Japanese and Chinese) were provided by Illumina (http://www.illumina. com/). Minor allele frequency data is not available for the Punjabi population. To eliminate the possibility that a dense marker map of 5858 SNPs would lead to an overestimation of $F$ due to linkage disequilibrium, three marker maps were evaluated: 5858 SNPs, intermarker distance $\sim 0.6 \mathrm{cM} ; 1170$ SNPs, intermarker distance $\sim 3 \mathrm{cM}$; and 585 SNPs, intermarker distance $\sim 6 \mathrm{cM}$. However, the lower marker density maps produced consistently higher inbreeding coefficients, suggesting that linkage disequilibrium does not contribute to inflated 
$F$ estimates. Inbreeding coefficients estimated using allele frequencies calculated for the Asian population were consistently higher at all marker densities than estimates calculated using CEPH population frequencies. Therefore, inbreeding coefficients presented in Table 1 reflect more conservative estimates generated using CEPH allele frequencies.

Analysis of homozygous SNPs and tracts across 22 autosomes in each individual was performed using PLINK v0.99 (http://pngu.mgh.harvard.edu/ purcell/plink) using 'Runs of homozygosity' analytical tool set. Inspection of homozygous tract lengths was limited to five or more consecutive SNPs, and low SNP densities in centromeric regions were excluded. The length of homozygous regions was taken to be from the most proximal to the most distal homozygous SNP, and the programme allows one heterozygous SNP within this run. Marker positions are based upon NCBI's build 35 physical map and decode genetic map.

\section{Sequencing of candidate genes}

Oligonucleotide primers were designed (http://workbench. sdsc.edu) for the coding regions and $500 \mathrm{bp}$ upstream (five prime UTR) and downstream (3 prime UTR) of KCNF1, ATF4 and CACNG2 genes. Due to extended size of annotated intronic sequences, these regions were not sequenced. Specificity of the primers for chromosome 2p25.1 and 22q13 was checked by blast analysis (http:// genome.ucsc.edu/ and http://www.ncbi.nlm.nih.gov). PCR amplification was performed using the Expand long template PCR system (Roche) and with a final volume of $20 \mu \mathrm{l}$ comprised of $25 \mathrm{ng}$ template DNA; $25 \mathrm{~mm}$ of each $\mathrm{dNTP} ; 5 \mu \mathrm{mol}$ primer; $1.7 \mu \mathrm{l} 10 \times$ PCR amplification buffer; and $0.25 \mathrm{U}$ Invitrogen Taq DNA polymerase. PCR conditions are described as follows: $95^{\circ} \mathrm{C}$ for $120 \mathrm{~s}, 32$ cycles consisting of $60 \mathrm{~s}$ at $94^{\circ} \mathrm{C}, 30 \mathrm{~s}$ at $54^{\circ} \mathrm{C}, 60 \mathrm{~s}$ at $72^{\circ} \mathrm{C}$, and the final extension step for $10 \mathrm{~min}$ at $72^{\circ} \mathrm{C}$. Sequencing was performed using the BigDye Terminator v31.1 Cycle Sequencing Kit and resolved on an ABI 377 sequencer. Sequence chromatograms were aligned using the phredPhrap software and visualized with the Consed program (http://bozeman.mbt.washington.edu/index.html). SNPs were identified and genotypes were noted by visual inspection of forward and reverse sequence traces.

\section{Results}

The microsatellite genome-wide scan revealed only one marker, D22S423, on chromosome 22q13 that was heterozygous for both parents and the unaffected offspring and homozygous for affected offspring. Fine mapping of the region by means of 19 additional markers revealed two further markers with the same pattern of HBD markers, D22S1178 and D22S1151, located 1.1 and 3.1 Mb telomeric to D22S423. Construction of parental haplotypes, illu- strated in Figure 2, indicated that D22S530 and D22S276 were also homozygous for the 'disease' alleles in all affected siblings and, importantly, either heterozygous or homozygous with one normal derived allele in non-affected parents. Consequently, three candidate regions define the boundaries of a 3.3 Mb disease locus (Figure 3). Assuming a recessive mode of inheritance, a maximum LOD score of 1.8 , at zero recombination fraction $\theta$ was calculated for D22S423, D22S1178 and D22S1151. The occurrence of intervals where markers are heterozygous in affected individuals situated between the closely spaced HBD regions could be explained by either double recombination events or mutation of the microsatellite markers. To determine which hypothesis is more accurate and simultaneously to fine-map the genome at a higher resolution, a second genome-wide scan using SNP markers was conducted.

The SNP data confirmed HBD on chromosome $22 \mathrm{q} 13$ with 15 markers covering a region of $\sim 12 \mathrm{Mb}$ displaying the HBD pattern. As indicated in Figure 3, the presence of intermittent non-HBD SNP markers gives rise to seven HBD candidate regions located between $35 \mathrm{Mb}$ (q12.3) and $47 \mathrm{Mb}$ (q13.3) on chromosome 22. Interestingly, the largest interval spanning $3 \mathrm{Mb}$ almost completely overlaps with the microsatellite-defined interval, suggesting that mutations within the microsatellites have occurred.

This putative disease interval houses a large number of genes and thus the choice of which genes to screen for mutations was based on functional candidacy. Activating transcription factor 4 (ATF4) located on 22q13.1 is a strong candidate gene for psychiatric illness. ATF4 interacts with DISC1, an established susceptibility factor associated with schizophrenia by multiple genetic and biological studies. ${ }^{12,13}$ A second strong candidate, CACNG2, encodes the protein stargazin, an L-type calcium channel subunit that also functions as a regulator of post-synaptic membrane targeting for AMPA type glutamate receptors. Intronic mutations in $C A C N G 2$ result in recessively inherited epilepsy and ataxia in stargazer mice; however, the introns are large and the exact position of some murine mutations is unknown. Furthermore, aberrations in DNA copy number in clones containing CACNG2 have been found in individuals with bipolar disorder and schizophrenia. ${ }^{14}$ Both these genes positioned on chromosome 22q12-3 within HBD intervals were screened in family members for mutations evident in affected individuals only. No obvious potential functional variants were identified in coding and promoter regions.

In addition to chromosome 22, the SNP data revealed a second interval on chromosome 2p24-25 that was homozygous in all affected family members and heterozygous in unaffected individuals. This region identified by markers rs730990-rs1978371 spans a distance of $\sim 5.4 \mathrm{Mb}$ (Figure 4), located $11 \mathrm{Mb}$ proximal to the pter. HBD was undetected in the first scan because the microsatellite 


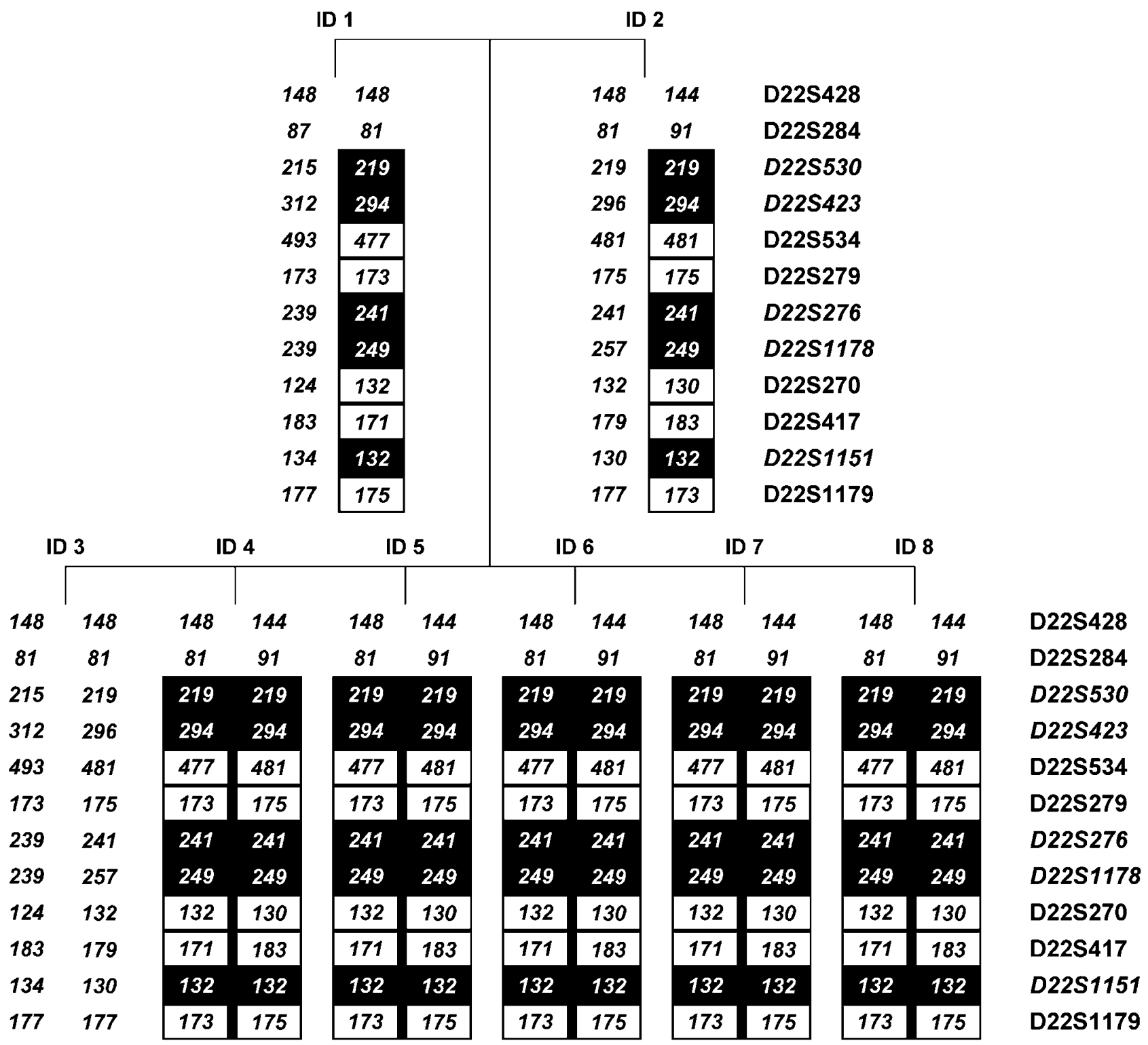

Figure 2 Constructed haplotypes of microsatellite markers on chromosome 22ql3. Identified disease haplotype is boxed. Homozygous by descent conforming markers are shaded black. Marker numbers denote microsatellite repeat genotype calls.

markers used are not positioned within this region. Candidate genes at 2p24.3-25.1 are relatively sparse. One gene of interest based on its function is KCNF1, a potassium voltage-gated channel of subfamily F. Mutations in voltage-gated potassium channel subunits have been found to be responsible for both monogenic epilepsies and heritable autosomal recessive syndromes such as Jervell and Lange-Nielsen syndrome, in which hearing impairment is a clinical feature. ${ }^{15,16}$ The promoter and coding regions of $K C N F 1$ was screened in all family members for mutations or functional variants. No such mutations were identified.
The inbreeding coefficients calculated using the 5858 SNP density map are presented in Table 1. As expected, $F$ values for the parents (ID 1: $0.136 \pm 0.027$; ID 2: $0.090 \pm 0.021$ ) are lower than all siblings (average $0.195 \pm 0.025$; range $0.16-0.22$ ). In addition, homozygous tracts across the 22 autosomes were analysed for each family member (Supplementary Material Graph S1). Homozygous runs evaluated were limited to five or more contiguous SNPs $\sim 2 \mathrm{Mb}$ in length, and hence short tracts of homozygosity were excluded from the data. Table 1 presents the average, medium and tract length range per individual. Tract length averages range from 5 to $7 \mathrm{Mb}$ and 
Homozygosity mapping in a consanguineous family

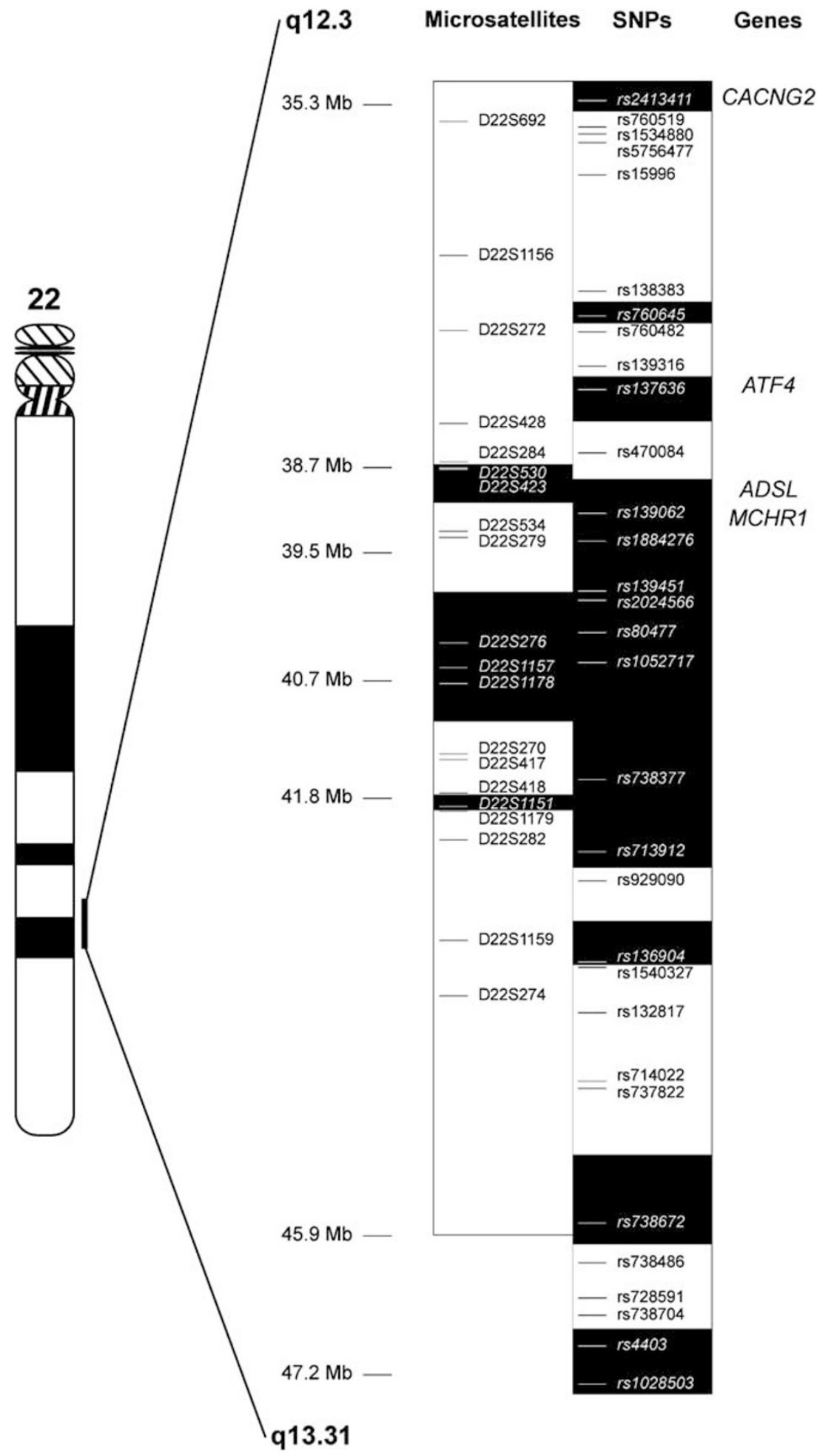

Figure 3 SNP and microsatellite data identifying locus on chromosome 22q12.3-13.3. HBD conforming markers shaded, non-HBD intervals not shaded. Physical distance along chromosome indicated. 
length medium from 2.97 to $4.08 \mathrm{Mb}$. The mother (ID 2) was found to have the shortest tracts, and a trend towards all offspring having longer average and medium tract lengths than both the parents is apparent. The longest homozygous tract for each individual ranged between 40

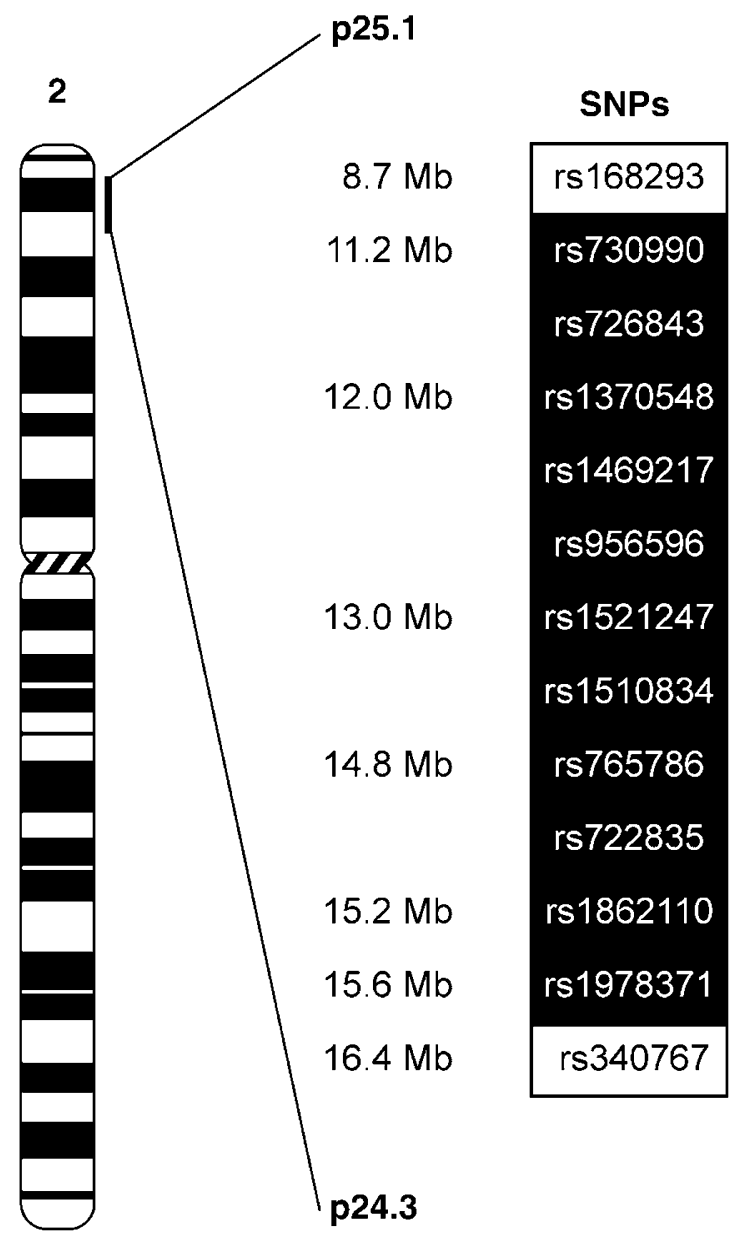

Figure 4 SNP markers identifying locus on chromosome 2p24.125.3. HBD conforming markers shaded black, non-HBD intervals white. and $74 \mathrm{Mb}$, with the most extensive being $74 \mathrm{Mb}$ found in individual 4 . The total number of tracts per individual (tract count) ranged between 92 and 114, with the parents having lower counts than their children. These data are consistent with the estimated $F$ values and indicate increased homozygosity in the offspring due to inbreeding over many generations.

\section{Discussion}

This study reports the mapping of two putative loci on chromosome $22 \mathrm{q} 13$ and 2p24-25 for a rare combination of schizophrenia, sensori-neural hearing impairment and epilepsy present in five out of six offspring of cousin parents. In this study, the parents had inbreeding coefficients calculated as higher than expected for offspring of first cousins, whereas the average value for the offspring (19\%) is greater than that predicted for double first cousins (12.5\%). These values, although very high, are still within the range of estimates (5-20\%) reported for the offspring of first-cousin marriages of Pakistani and Arabian descent ${ }^{17}$ and are in keeping with our knowledge that, historically, marriages within this family have been restricted to a small group of individuals from a small geographical region.

In the aforementioned study of 48 affected individuals with diverse autosomal recessive diseases born of firstcousin marriages and of Pakistani and Arabian descent, an average 20 homozygous segments exceeding $3 \mathrm{cM}$ in size and an average tract size of $19.5 \mathrm{cM}$ per affected person was estimated. This data estimate an average tract size for offspring $\sim 6.58 \pm 0.44 \mathrm{Mb}$, and average tract count $111 \pm 5$ (SEM). Assuming parity between $\mathrm{cM}$ and $\mathrm{Mb}$, this would suggest a fivefold greater number of tracts that are, on average, a fifth of the size than that reported by Woods et $a l^{17}$ - a difference that may reflect more remote ancestry through additional ancestral inbreeding loops in this family.

In this study, we have carried out analysis based on a recessive model assuming that this is a single rare phenotype that includes features of hearing loss, epilepsy

Table 1 Analysis of homozygosity tract lengths and inbreeding coefficients per individual

\begin{tabular}{|c|c|c|c|c|c|}
\hline Family Individual & Average $(\mathrm{Mb})$ & Median $(\mathrm{Mb})$ & Range (Mb) & No. of tracts & Inbreeding coefficient (F) \\
\hline ID 1 & $6.52($ SD 9.3) & 3.30 (IQR 2.2-5.8) & $0.7-55.2$ & 100 & 0.136 (SD 0.027) \\
\hline ID 2 & 5.00 (SD 6.0) & 2.97 (IQR $2.1-5.2)$ & $0.5-46.6$ & 92 & 0.090 (SD 0.021) \\
\hline ID 3 & 6.00 (SD 7.4) & 3.62 (IQR 2.4-5.6) & $0.1-39.3$ & 119 & 0.161 (SD 0.260) \\
\hline ID 3 & 6.52 (SD 9.1) & 4.08 (IQR $1.8-6.7)$ & $0.3-73.7$ & 112 & 0.205 (SD 0.027$)$ \\
\hline ID 4 & 6.89 (SD 9.2) & 3.49 (IQR 2.4-6.7) & $0.1-53.8$ & 108 & 0.186 (SD 0.028) \\
\hline ID 5 & 6.18 (SD 7.9) & 3.98 (IQR 2.4-6.0) & $0.3-60.2$ & 114 & 0.175 (SD 0.025) \\
\hline ID 6 & 6.72 (SD 8.5) & 3.97 (IQR 2.7-6.4) & $0.1-61.3$ & 106 & 0.222 (SD 0.029) \\
\hline ID 7 & 7.16 (SD 9.4) & 3.76 (IQR 2.3-6.3) & $0.1-55.0$ & 106 & 0.222 (SD 0.031) \\
\hline
\end{tabular}

Note: Analysis of homozygous segments was limited to five or more contiguous SNPs in a tract. Hence, the minimum value presented for range represents shortest tract length of five contiguous SNPs. 
and schizophrenia. A link between deafness and schizophrenia has been described in the literature particularly in old age. ${ }^{18}$ However, it is unlikely that the schizophrenia phenotype in the family is a direct result of deafness, as the onset is early and the symptoms of psychosis are episodic with good recovery between episodes. Furthermore, we argue that mutations in one or more genes located in the identified $\mathrm{HBD}$ regions interact with environmental or other genetic factors, giving rise to the variable penetrance/ expressivity of clinical features. Nevertheless, other modes of inheritance should be considered. For example, dominant inheritance with variable penetrance and mitochondrial inheritance can indeed give rise to phenotypic manifestations similar to that of this family (eg MELAS and MERRF disorders). ${ }^{19}$ However, the existence of inbreeding and the lack of cases identified with the phenotype from the same region in Punjab do not support these alternative models.

Deletions and duplications can disturb the expected patterns of marker genotypes within pedigrees. A common deleted segment on one chromosome in both parents would result in missing genotype data in all siblings that inherit this deletion (ie null genotypes). Likewise, SNP genotyping failures may indicate commonly inherited duplicated regions. ${ }^{20}$ SNP marker analysis did not reveal any single SNP or regions of missing data in affected siblings, nor were there any Mendelian inconsistencies identified at the resolution of our marker density ( $\sim 480 \mathrm{~kb})$. Therefore, although the phenotype is consistent with a contiguous gene syndrome, no gross duplications or deletions are evident.

A limitation of homozygosity mapping, as for linkage studies of small families, is that it detects large disease intervals that may house hundreds of genes. Although fine mapping or previous linkage reported in other families may reduce the size of the putative interval, the overall aim of such studies, gene identification, is dependent on examining functional candidate genes in the region. With regard to hereditary sensori-neural hearing impairment, the recently documented DFNB47 locus is linked to autosomal recessive hearing impairment and maps to a 5.3-Mb-sized region on $2 \mathrm{p} 24.3-25.1 .^{21}$ Of interest, the maximum LOD score of 4.7 is reported for markers D2S1400 and D2S262, both of which are located within our chromosome $2 \mathrm{HBD}$ region. The two large pedigrees segregating this locus live in Pakistan, but are geographically distant from the family described, and there is no suggestion that the families will be related. As in this study, the candidate gene, KCNF1, was screened in affected individuals in these two large kindreds and the results were also negative.

Findings from linkage studies provide strong evidence that a single major gene on chromosome $22 \mathrm{q} 12$ is responsible for an idiopathic epilepsy syndrome. Familial partial epilepsy syndrome with variable foci (FPEVF), an autosomal dominant and clinically heterogeneous condition, was initially mapped in two large French-Canadian pedigrees to a $\sim 4 \mathrm{Mb}$ interval on chr 22q11-12. ${ }^{22}$ Two preceding studies confirmed linkage for FPEVF in Dutch, Spanish and French-Canadian pedigrees reporting a combined LOD score of 6.3 for the Spanish and three

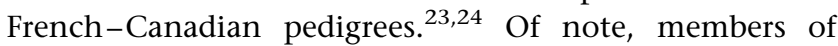
these pedigrees are reported to have psychiatric problems including the diagnosis of paranoid schizophrenia. Although not overlapping with this study's disease interval on $22 \mathrm{q} 13$, the most telomeric region of the FPEVF locus is just $\sim 4 \mathrm{Mb}$ from our HBD region.

Epidemiological and clinical characteristics common to schizophrenia and bipolar disorder suggest that the two disorders share genetic and non-genetic susceptibility factors. ${ }^{25}$ Over the last decade, converging evidence from independent studies strongly implicate chromosome $22 \mathrm{q}$ as a candidate region for susceptibility genes for these disorders. Positive findings, as reported in 1999, cluster in a region of $4-5 \mathrm{cM}$ on $22 \mathrm{q} 13.1$, an interval mapping directly to our 'core' region between markers D22S284 and D22S270. ${ }^{26}$ More recent linkage analysis of markers on $22 \mathrm{q}$ for schizophrenia and bipolar disorder fulfils the criteria of suggestive or significant linkage, implicating a broad genomic region spanning 22q12.3-13.3 that coincides with the findings of this study. ${ }^{27,28}$

The screening of the promoter and coding regions of ATF4 and CACNG2 did not reveal any apparent mutations in the affected family members. It is still possible that mutations in intronic regulatory regions of these genes underlie this recessive disease. Furthermore, several alternative candidate genes for this specific phenotype are located on chromosome 22q13 (Figure 3). For example, $A D S L$ encodes the protein adenylsuccinate lyase, a deficiency that causes a selectively neuronopathic disorder with psychomotor retardation, epilepsy and autistic-like features as leading traits. ${ }^{29}$ Moreover, $A D S L$ and the promoter region of the neighbouring gene $G$ proteincoupled receptor 24 (MCHR1 or alternative nomenclature GPR24) were found to be significantly associated with both SZ and BPD in Scottish and Faeroe Island disease populations. ${ }^{30,31}$ Mutation screening of these and other promising candidate genes within 22q12-3-13.3 and 2p24.3-25.1 may lead to the discovery of causative mutations associated with this rare phenotype.

\section{Acknowledgements}

We are grateful to the members of the family who have given their full consent to these studies. We thank Professor Alan Wright and Dr Caroline Hayward at the Medical Research Council Human Genetics Unit, Western General Hospital, Edinburgh, for their advice and assistance with the analysis of homozygous tracts and to Anne-Louise Leutenegger for discussion and the program FEstim. This work was funded by a grant from the Chief Scientist Office of the Scottish Executive. 


\section{Financial Disclosures}

All authors reported no biomedical financial interests or potential conflicts of interest.

\section{References}

1 Sheffield VC, Stone EM, Carmi R: Use of isolated inbred human populations for identification of disease genes. Trends Genet 1998; 14: 391-396.

2 Lander ES, Botstein D: Homozygosity mapping: a way to map human recessive traits with the DNA of inbred children. Science 1987; 236: 1567-1570.

3 Petit C: Genes responsible for human hereditary deafness: symphony of a thousand. Nat Genet 1996; 14: 385-391.

4 Van Camp G, Smith R: The hereditary hearing loss homepage http://www.webhost.ua.ac.be/hhh/, 2005.

5 Piatto VB, Nascimento EC, Alexandrino F et al: Molecular genetics of non-syndromic deafness. Rev Bras Otorrinolaringol (Engl Ed) 2005; 71: 216-223.

6 Mulley JC, Scheffer IE, Harkin LA, Berkovic SF, Dibbens LM: Susceptibility genes for complex epilepsy. Hum Mol Genet 2005; 14 (Spec No. 2): R243-R249.

7 Macgregor S, Visscher PM, Knott S et al: Is schizophrenia linked to chromosome 1q? Science 2002; 298: 2277; author reply 2277.

8 Millar JK, Pickard BS, Mackie S et al: DISC1 and PDE4B are interacting genetic factors in schizophrenia that regulate cAMP signaling. Science 2005; 310: 1187-1191.

9 Terwilliger JD, Ott J: Handbook of Human Genetic Linkage. Baltimore: The John Hopkins University Press, 1994.

10 Leutenegger AL, Prum B, Genin E et al: Estimation of the inbreeding coefficient through use of genomic data. Am J Hum Genet 2003; 73: 516-523.

11 Leutenegger AL, Labalme A, Genin E et al: Using genomic inbreeding coefficient estimates for homozygosity mapping of rare recessive traits: application to Taybi-Linder syndrome. Am J Hum Genet 2006; 79: 62-66.

12 Millar JK, Wilson-Annan JC, Anderson S et al: Disruption of two novel genes by a translocation co-segregating with schizophrenia. Hum Mol Genet 2000; 9: 1415-1423.

13 Morris JA, Kandpal G, Ma L, Austin CP: DISC1 (disrupted-inschizophrenia 1) is a centrosome-associated protein that interacts with MAP1A, MIPT3, ATF4/5 and NUDEL: regulation and loss of interaction with mutation. Hum Mol Genet 2003; 12: 1591-1608.

14 Wilson GM, Flibotte S, Chopra V, Melnyk BL, Honer WG, Holt RA: DNA copy-number analysis in bipolar disorder and schizophrenia reveals aberrations in genes involved in glutamate signaling. Hum Mol Genet 2006; 15: 743-749.

15 Schwartz PJ, Spazzolini C, Crotti L et al: The Jervell and LangeNielsen syndrome: natural history, molecular basis, and clinical outcome. Circulation 2006; 113: 783-790.
16 Jervell A, Lange-Nielsen F: Congenital deaf-mutism, functional heart disease with prolongation of the Q-T interval and sudden death. Am Heart J 1957; 54: 59-68.

17 Woods CG, Cox J, Springell K et al: Quantification of homozygosity in consanguineous individuals with autosomal recessive disease. Am J Hum Genet 2006; 78: 889-896.

18 Cooper AF: Deafness and psychiatric illness. Br J Psychiatry 1976; 129: $216-226$.

19 Simon DK, Johns DR: Mitochondrial disorders: clinical and genetic features. Annu Rev Med 1999; 50: 111-127.

20 Redon R, Ishikawa S, Fitch KR et al: Global variation in copy number in the human genome. Nature 2006; 444: 444-454.

21 Hassan MJ, Santos RL, Rafiq MA et al: A novel autosomal recessive non-syndromic hearing impairment locus (DFNB47) maps to chromosome 2p25.1-p24.3. Hum Genet 2006; 118: $605-610$

22 Xiong L, Labuda M, Li DS et al: Mapping of a gene determining familial partial epilepsy with variable foci to chromosome 22q11q12. Am J Hum Genet 1999; 65: 1698-1710.

23 Callenbach PMC, van den Maagdenberg AMJM, Hottenga JJ et al: Familial partial epilepsy with variable foci in a Dutch family: clinical characteristics and confirmation of linkage to chromosome 22q. Epilepsia 2003; 44: 1298-1305.

24 Berkovic SF, Serratosa JM, Phillips HA et al: Familial partial epilepsy with variable foci: clinical features and linkage to chromosome 22q12. Epilepsia 2004; 45: 1054-1060.

25 Berrettini WH: Are schizophrenic and bipolar disorders related? A review of family and molecular studies. Biol Psychiatry 2000; 48: 531-538.

26 Schwab SG, Wildenauer DB: Chromosome 22 workshop report. Am J Med Genet 1999; 88: 276-278.

27 Jorgensen $\mathrm{TH}$, Borglum AD, Mors $\mathrm{O}$ et al: Search for common haplotypes on chromosome $22 \mathrm{q}$ in patients with schizophrenia or bipolar disorder from the Faroe Islands. Am J Med Genet 2002; 114: $245-252$.

28 Kelsoe JR, Spence MA, Loetscher $\mathrm{E}$ et al: A genome survey indicates a possible susceptibility locus for bipolar disorder on chromosome 22. Proc Natl Acad Sci USA 2001; 98: 585-590.

29 Race V, Marie S, Vincent MF, Van den Berghe G: Clinical, biochemical and molecular genetic correlations in adenylosuccinate lyase deficiency. Hum Mol Genet 2000; 9: $2159-2165$

30 Severinsen JE, Bjarkam CR, Kiaer-Larsen $\mathrm{S}$ et al: Evidence implicating BRD1 with brain development and susceptibility to both schizophrenia and bipolar affective disorder. Mol Psychiatry 2006; 11: 1126-1138.

31 Severinsen JE, Als TD, Binderup $\mathrm{H}$ et al: Association analyses suggest GPR24 as a shared susceptibility gene for bipolar affective disorder and schizophrenia. Am J Med Genet B Neuropsychiatr Genet 2006; 141: 524-533.

Supplementary Information accompanies the paper on European Journal of Human Genetics website (http://www.nature.com/ejhg) 